\title{
EDITORIAL
}

\section{Impact assessment of landfalling tropical cyclones: introduction to the special issue}

\author{
Hui YU (凶) ${ }^{1}$, Lianshou CHEN ${ }^{2}$ \\ 1 Shanghai Typhoon Institute, China Meteorological Administration, Shanghai 200030, China \\ 2 Chinese Academy of Meteorological Sciences, Beijing 100081, China
}

(C) Higher Education Press and Springer-Verlag GmbH Germany, part of Springer Nature 2019

\section{Introduction}

Tropical cyclones (TCs) are one of the most destructive natural phenomena in the world. The accompanied damaging winds, heavy precipitation, and storm surges have caused great economic losses and life casualties to human beings. There are eight disaster events related to TC among the top tenwhich caused the highest amount of insurance losses in the world.

Frequency of TC genesis in the western North Pacific is the highest in the global ocean basins, and there are nine on average making landfall in China annually. Most of these landfalling TCs (LTCs) strike the south-east coastal region of China with a dense population and developed economies. Some of them intrude deep inland or higherlatitude and cause severe flooding. The most recent example is Super Typhoon Lekima, which made landfall in east China on 10 August 2019. Lekima brought severe winds and precipitation to the Eastern and North-eastern China, from Fujian province all the way north to Heilongjiang province. The observed maximum wind is $61.4 \mathrm{~m} / \mathrm{s}$ near the landfall point, and the maximum total precipitation reaches $831 \mathrm{~mm}$.

Chinese governments at all levels and China Meteorological Administration have always attached great importance to TC disaster prevention and reduction. Two five-year National Basic Research Program of China (973 Program) (Nos. 2009CB421500 and 2015CB452806) projects on LTCs were launched by the Ministry of Sciences and Technology in 2009 and 2015, respectively. The impact assessment of LTCs is one of the research focal points for both projects. Shanghai Typhoon Institute takes the lead in that part of the research for a consecutive of ten years in close collaboration with Chinese Academy of Meteorological Sciences. Significant progress has been made in TC disaster risk zoning at county levels, TC forecast uncertainty analyses, TC impact assessment using remote sensing technique, pre-assessment of LTC-related engineering damages, landslides potential, and inundation risk at a high resolution up to meters or tens of meters, and so on.

This special issue of Frontiers of Earth Science is initiated by Shanghai Typhoon Institute in the final year of the implementation period of the second 973 Program (No. 2015CB452806), with an aim to collect research articles which provide further insight into the activities of TCs and the developments in methods that are being used to assess the impact of LTCs. A total of 15 peer-reviewed papers are selected for publication at a variety of topics related to LTCs, including TC impact assessment index development, new remote sensing exploring techniques, new forecast techniques, fine structure and abrupt intensity change analyses, observation and forecast uncertainty analyses, and so on.

\section{Overview of special issue papers}

The 15 papers in this special issue are divided into four topics: 1) impact of precipitation and wind from LTC; 2)

Received October 11, 2019; accepted November 14, 2019

E-mail: yuh@typhoon.org.cn 
forecast of LTC; 3) track, intensity and structure of LTC; 4) others. There are 4, 4, 5, and 2 papers under the four topics, respectively.

\subsection{Impact of precipitation and wind from LTC}

An index, named IPWT, was proposed by Chen et al. (P672-P681) to assess the severity of the combined impact of precipitation and wind from TC. A five-category scaling system was set up based on IPWT for TCs affecting China, namely Category $0,1,2,3$, and 4 . The higher the category, the greater the losses from the impact of TC. Such an index, by including both the precipitation and wind factors, is a good supplement to the traditional categorizing system of TC based only on the maximum surface wind speed near the center.

Wu et al. (P682-P694) proposed workflow in Google Earth Engine for mapping the flooding and lodging area of paddy rice after Super Typhoon Maria (2018). Change Detection based Rice Normalized Difference Flooded Index $(R N D F I)$ and Rice Normalized Difference Lodged Index ( $R N D L I)$ were proposed to detect flooding and lodged paddy rice. Tests using four different remote sensing data sets showed very good agreement in flood and lodging detection.

Chen et al. (P695-P704) examined the parameter sensitivity of the landslide prediction from two physical models to the uncertainty in rainfall data. The Transient Rainfall Infiltration and Grid-based Regional Slope Stabilityprobabilistic model (TRIGRS-P) adopts a probabilistic approach to compute the changes in the Factor of Safety due to rainfall infiltration. The Slope Infiltration Distributed Equilibrium model is modified by adopting the same probabilistic approach (SLIDE-P), which allows values of the model input parameters to be sampled randomly. The probabilistic prediction from TRIGRS-P model was found to be more concentrated than that from SLIDE-P model when using different rainfall forcing data.

Fang et al. (P705-P720) introduced a simplified two-dimensional (2D) terrain parameterization scheme, which had the potential to be used in diagnostic wind field downscaling models for LTC. Aerodynamic characteristics of the $2 \mathrm{D}$ terrain were investigated with both the wind tunnel experiments and numerical model simulations. Special attention was paid to how the slope angle could affect the wind pressure coefficients and the wind speed ratios.

\subsection{Forecast of LTC}

A micro-genetic algorithm was applied to optimize the parameters of Kain-Fritsch convective parameterization scheme, including the convective time scale and the conversion rate (Zhu et al., P721-P732). The aim was to improve the quantitative precipitation forecast (QPF) of LTC. The optimizing process was controlled by the microgenetic algorithm using a QPF skill score as the fitness function. Two case studies demonstrated the effectiveness of the method in improving the QPF of LTC.

A Typhoon Ensemble Data Assimilation and Prediction System (TEDAPS) was developed by Li et al. (P733P743) using an ensemble-based data assimilation scheme and a multi-physics approach. A 4-year evaluation demonstrated that TEDAPS is good at intensity forecast by effectively reducing the weak biases and is capable of capturing the track forecast uncertainty, showing a great potential to be used in operation.

Huang et al. (P744-P757) investigated the impact of physical representations in a diagnostic wind field model CALMET on simulations of the near-surface wind field of Super Typhoon Meranti (2016). It was found that the blocking effects could exceed $10 \mathrm{~m} / \mathrm{s}$ before the landfall of Meranti in a mountainous region, but were very weak in the strong wind area after Meranti made landfall. The slope flows were generally smaller than $3 \mathrm{~m} / \mathrm{s}$ no matter whether the area was affected by strong winds or not.

A probabilistic forecast scheme was developed by Zhao et al. (P758-P777) for the precipitation in LTC. They used the Bayesian Model Averaging method to calibrate the precipitation forecast from three ensemble prediction systems (EPSs). An improvement of $12.4 \%$ was obtained in the mean absolute error of $24 \mathrm{~h}$ forecast.

\subsection{Track, intensity and structure of LTC}

Liu et al. (P778-P790) studied the impacts of multi-time-scale flows on northward and north-eastward moving TCs near the east coast of China. Decomposition analyses showed that the meridional steering vector was dominated by the intraseasonal component, while the eastward zonal component was mainly contributed by the climatological mean flow.

Tang et al. (P791-P807) studied the rapid intensification (RI) process of Super Typhoon Meranti (2016) using a cloud-resolving model. They found that the inner core convections took mode turns from stratiform-precipitation dominance to convective-precipitation prevalence during the transition stages between pre-RI and RI. There was a 
clear upper-level warm core structure during RI, and the link between convections and the warm core was established in the paper.

Ji et al. (P808-P816) examined the environmental characteristics of LTCs experiencing abrupt intensity change. Favorable conditions for RI include warm sea surface temperature, sufficient water vapor transportation, and moderate low-level relative vorticity.

Jiao et al. (P817-P828) studied the evolution of hollow potential vorticity (PV) tower extending from the lower troposphere to the middle levels in Typhoon Mujigae (2015) during its landfall process. Diagnostics on the PV budget revealed the importance of symmetric heating in the generation and maintenance of the hollow PV tower.

A fuzzy logic algorithm was developed by Cheng et al. (P829-P835) to discriminate cloud type based on millimeter-wave cloud radar observations. A total of 8 cloud types can be discriminated by the algorithm. An experiment with Typhoon Khanun (2017) demonstrated the effectiveness of the algorithm in revealing changes in cloud type of the LTC.

\subsection{Others}

A spiral cloud belt matching (SCBeM) technique was proposed by Lu et al. (P836-P847) to locate the TC center automatically using geo-satellite images. Independent experiments showed that the technique had a median error of about $50 \mathrm{~km}$ and performed better for strong TCs than it does for weak ones. It is not suitable for TCs at high-latitudes or over land when their spiral cloud bands were destroyed by westerlies or terrain.

Fang et al. (P848-P858) investigated numerically the wind environment among main buildings in a central business district (CBD) in Shanghai. Typical flow patterns were identified reasonably among buildings, such as the stagnation point and shielding effect, separation and channeling flow. Some pedestrian activities were found to be unsafe when the wind blows from the south-east direction, such as sitting at the center of the three tallest buildings.

We would like to express special thanks to Prof. Shu Jiong (East China Normal University), Prof. Wu Liguang (Fudan University), and Prof. Li Xiaofan (Zhejiang University) for their generous and professional contributions to the special issue as the guest editors.

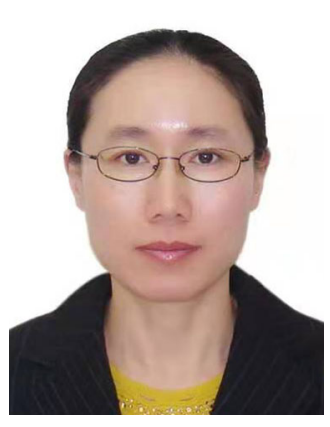

Dr. Hui Yu is the director of Shanghai Typhoon Institute (STI) of China Meteorological Administration, a member of the Working Group on Tropical Meteorological Research under World Meteorological Organization (WMO), and the deputy-chief-editor of the UNESCAP/WMO Typhoon Committee journal Tropical Cyclone Research and Review. Her research interests include tropical cyclone track, intensity, and structure change, tropical cyclone analyses with observations from multiple sources, interaction between tropical cyclones and mid-latitude weather systems, tropical cyclone prediction techniques, climatology and disaster risk assessment.

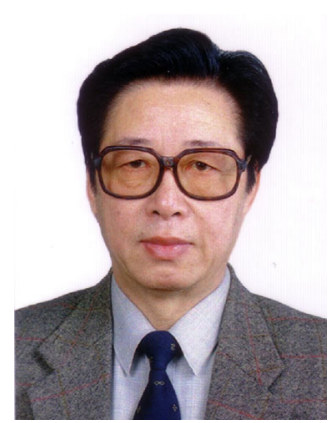

Prof. Lianshou Chen is an Academician of the Chinese Academy of Engineering, (CAE) and researcher of Chinese Academy of Meteorological Sciences. He used to be the visiting scholar of Colorado state University, USA (1982 - 1984), director of Central Meteorological Observatory, president of Chinese Academy of Meteorological Sciences, vice Chairperson of council on Chinese Meteorological Society, the chief scientist of research project on TOPEX and the second atmospheric scientific field experiment on Tibet Plateau, the Chairperson of Working Group on Tropical Meteorological Research under World Meteorological Organization (WMO) (2002-2010). 